\title{
Field Emission Properties of the Graphene Double-Walled Carbon Nanotube Hybrid Films Prepared by Vacuum Filtration and Screen Printing
}

\author{
Jinzhuo Xu, ${ }^{1}$ Tao Feng, ${ }^{1,2}$ Yiwei Chen, ${ }^{1}$ and Zhuo Sun ${ }^{1}$ \\ ${ }^{1}$ Engineering Research Center for Nanophotonics \& Advanced Instrument, Ministry of Education, Department of Physics, \\ East China Normal University, 3663 North Zhongshan Road, Shanghai 200062, China \\ ${ }^{2}$ Herbert Gleiter Institute of Nanoscience, Nanjing University of Science \& Technology, 200 Xiaolinwei Road, \\ Nanjing 210094, China
}

Correspondence should be addressed to Tao Feng; tao.feng@njust.edu.cn

Received 26 September 2013; Accepted 25 October 2013

Academic Editor: Hui Xia

Copyright (C) 2013 Jinzhuo Xu et al. This is an open access article distributed under the Creative Commons Attribution License, which permits unrestricted use, distribution, and reproduction in any medium, provided the original work is properly cited.

\begin{abstract}
The graphene double-walled carbon nanotube (DWCNT) hybrid films were prepared by vacuum filtration and screen printing. Their electron field emission properties have been studied systematically. The electron emission properties of the hybrid films are much better than those of pure DWCNT films and pure graphene films. Comparing with the screen printed films, the vacuum filtered films have many advantages, such as lower turn-on field, higher emission current density, better uniformity, better longterm stability, and stronger adhesive strength with conductive substrates. The optimized hybrid films with $20 \%$ weight ratio of graphene, which were fabricated by vacuum filtration, show the best electron emission performances with a low turn-on field of $0.50 \mathrm{~V} \mu \mathrm{m}^{-1}\left(\right.$ at $\left.1 \mu \mathrm{Acm}^{-2}\right)$ and a high field enhancement factor $\beta$ of 27000 .
\end{abstract}

\section{Introduction}

Field emission relies on the electron extraction from the material surface by quantum mechanical tunneling [1]. This simple principle has been widely used in field emission displays (FED) [2], electron guns [3, 4], back-light devices [5], and so on.

Due to their high aspect ratio, nanometer-sized tip radii, low work function, and high electrical conductivity, carbon nanotubes (CNTs) are considered one of the most promising materials for electron field emission [6-12]. However, the integration of CNT field emission into device remains a challenge which stems from the method of preparing field emission films and the contact between CNT films and the electrical substrates.

Lots of methods for fabricating CNT field emission films are developed. CNT films prepared by the chemical vapor deposition (CVD) method [13-15] have high conductivity. But this method is very complex and expensive. Screen printing [16] is widely used to fabricate CNT field emission films, due to its low cost, easy control, and scalable preparation; however, the simple screen printing technique also brings some drawbacks, such as the poor adhesive strength to the substrate, residual organic binders, and high annealing temperature. The electrophoresis method [17] for fabricating CNT films owes some disadvantages, like the bad uniformity of films and the poor adhesive strength to the substrate. Vacuum filtration [18] is also widely used to prepare CNT films, owing to its low cost, being scalable, and low temperature preparation. Films prepared by vacuum filtration have the advantages of no organic binders and strange adhesive strength to the substrate.

On the other hand, graphene a two-dimensional (2D) carbon material owns the highest electron mobility of $15,000 \mathrm{~cm}^{2}$ 
$(\mathrm{V} \cdot \mathrm{cm})^{-1}$ at room temperature [19-23], which is currently of great interest as efficient FE sources because of its unique electronic properties, large surface areas, and sharp edges [24, 25]. However, the existing preparation methods always lead to graphene that has the planar morphological features along the entire substrates, which limits the field enhancement. To overcome this problem, a hybrid structure of 1D DWCNTs and $2 \mathrm{D}$ graphene films was prepared in this paper. The graphene-DWCNT hybrid films fabricated by screen printing and vacuum filtration were proposed to improve the field emission properties. We compared the field emission performances of the graphene-DWCNT hybrid films fabricated by screen printing and vacuum filtration. Our results show that adding a certain amount of graphene in the DWCNT films will improve the field emission performance, and the field emission performances of graphene-DWCNT hybrid films fabricated by vacuum filtration are better than those of films fabricated by screen printing.

\section{Experimental Section}

2.1. Preparation of Graphene by Chemical Reduction. Graphene was prepared by the reduction of graphene oxide (GO), and GO was prepared from natural graphite by modified hummer method [21]. The natural graphite ( $3 \mathrm{~g})$ was purified by mixed acid (sulfuric acid $(12 \mathrm{~mL})$ and nitric acid $(24 \mathrm{~mL})$ ) at $80^{\circ} \mathrm{C}$. The purified graphite was added in $120 \mathrm{~mL}$ sulfuric acid. Then, the mixture was cooled down to $0^{\circ} \mathrm{C}$ in ice bath, and this was followed by carefully adding $12 \mathrm{~g}$ potassium hypermanganate. Oxidizing reaction was taken in an oil bath at $35^{\circ} \mathrm{C}$ for 2 hours. After the oxidizing process, $750 \mathrm{~mL}$ deionized water (DI-water) was added in drop by drop. After a 2-hour stirring, $10 \mathrm{~mL}$ hydrogen peroxide was added in the mixture, and the solution became yellow. And then, after centrifuging the dispersion for $15 \mathrm{~min}$ and drying it at $60^{\circ} \mathrm{C}$, the GO powder was prepared.

The as-prepared GO (1g) powder was dissolved in DI water $(1000 \mathrm{~mL})$, and then $500 \mu \mathrm{L}$ hydrazine was added into the solution. The reduction was held for $12 \mathrm{~h}$ with a temperature of $80^{\circ} \mathrm{C}$. After vacuum filtering and being dried at $60^{\circ} \mathrm{C}$ for $12 \mathrm{~h}$, graphene was prepared.

\subsection{Preparation of Field Emission Cathode by Vacuum Fil-} tration. The as-prepared graphene and DWCNT (Shenzhen Nanotech Port Co., Ltd., purity: CNTs > 90\%, diameter < $5 \mathrm{~nm}$, length of $5-15 \mu \mathrm{m})$ mixed powder $(50 \mathrm{mg})$ with different weight ratios of graphene and sodium dodecyl sulfate (SDS) $(50 \mathrm{mg})$ was dissolved in DI water $(100 \mathrm{~mL})$ aided by a $30 \mathrm{~min}$ ultrasonic dispersion. The graphene-DWCNT hybrid solution $(50 \mathrm{~mL})$ was filtered through a $0.22 \mu \mathrm{m}$ mixed cellulose ester (MCE) membrane (diameter of $47 \mathrm{~mm}$ ) and then washed with DI water to remove SDS. The grapheneDWCNT hybrid films which are kept flat on the MCE membrane can be transferred to metal substrates or flexible substrates. We transferred the hybrid films to glass plates coated with silver by dissolving the MCE membrane with a repeated bath of acetone vapor. We put the hybrid films on the electric substrates directly in nylon/hybrid films/electric substrate structure in the acetone bath. After the clean wash, the upper nylon film can be removed easily. Then, the graphene-DWCNT films adhered tightly to the conductive substrate. At last, the sample was dried at $80^{\circ} \mathrm{C}$ for 3 hours to remove acetone vapor. An adhesive tape method has been taken to improve the field emission performance [26].

\subsection{Preparation of Field Emission Cathode by Screen Printing.} The composite powder of graphene and DWCNTs with different weight ratios $(0.2 \mathrm{~g})$ mixed with ethyl cellulose and terpineol $(1.4 \mathrm{~g})$ was screen-printed onto silver coated substrates $(1 \mathrm{~cm} \times 1 \mathrm{~cm})$. These samples were dried at $200^{\circ} \mathrm{C}$ for $10 \mathrm{~min}$ and then annealed at $300^{\circ} \mathrm{C}$ to burn out organic binders. An adhesive tape method has been taken to improve the field emission performance.

2.4. Characterization of Samples. The morphology of screenprinted graphene-DWCNT hybrid cathode was examined by field emission scanning electron microscopy (FESEM) using HITACHI S-4800 at a working voltage of $5.0 \mathrm{KV}$. Field emission properties were performed in vacuum $(6.5 \times$ $10^{-4} \mathrm{~Pa}$ ) by a homemade conventional diode-type structure. A $340 \mu \mathrm{m}$ thick glass spacer was used to keep the distance between cathode and anode.

\section{Results and Discussion}

The field emission performances of graphene-DWCNT hybrid films with different weight ratios of graphene in DWCNTs and with different preparation methods are systematically studied. The emission current density versus applied electric field of different samples with vacuum filtration and screen printing is shown in Figures 1(a) and 1(b). The weight ratio of graphene and fabrication method are clearly shown in the label of every sample such that VFGC-20 means that the sample is prepared by vacuum filtration and the weight ratio of graphene is $20 \%$. All samples in each figure can be divided into three parts as the weight ratio of graphene increases from $0 \%$ to $100 \%$. In the first group, when the weight ratio of graphene is less than $20 \%$, the field emission performance turns better as the weight ratio of graphene increased. In the second group, when the ration of graphene is between $20 \%$ and $40 \%$, the field emission performance turns worse, but the FE performance is still better than that of the pure DWCNT films. In the last group, when the weight ratio of graphene is bigger than $40 \%$, the field emission performance turns worse when increasing the weight ratio of graphene, and the field emission performance is also worse than that of DWCNTs.

The field emission data of Figures 1(a) and 1(b) follows approximately the well-known Fowler-Nordheim (F$\mathrm{N}$ ) equation which is widely used in carbon based electron emitters for the analysis of field emission enhancement factor $\beta$. The field enhancement factor can be calculated using the $\mathrm{F}-\mathrm{N}$ equation as follows:

$$
J=A\left(\frac{\beta^{2} E^{2}}{\Phi}\right) \exp \left(\frac{-B \Phi^{1.5}}{\beta E}\right)
$$




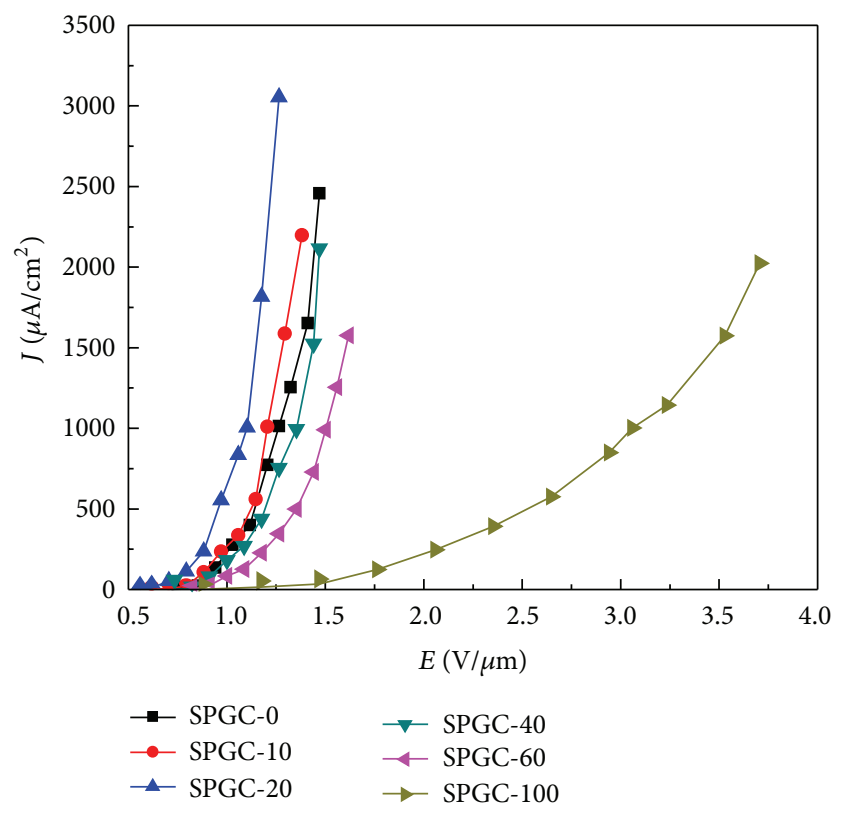

(a)

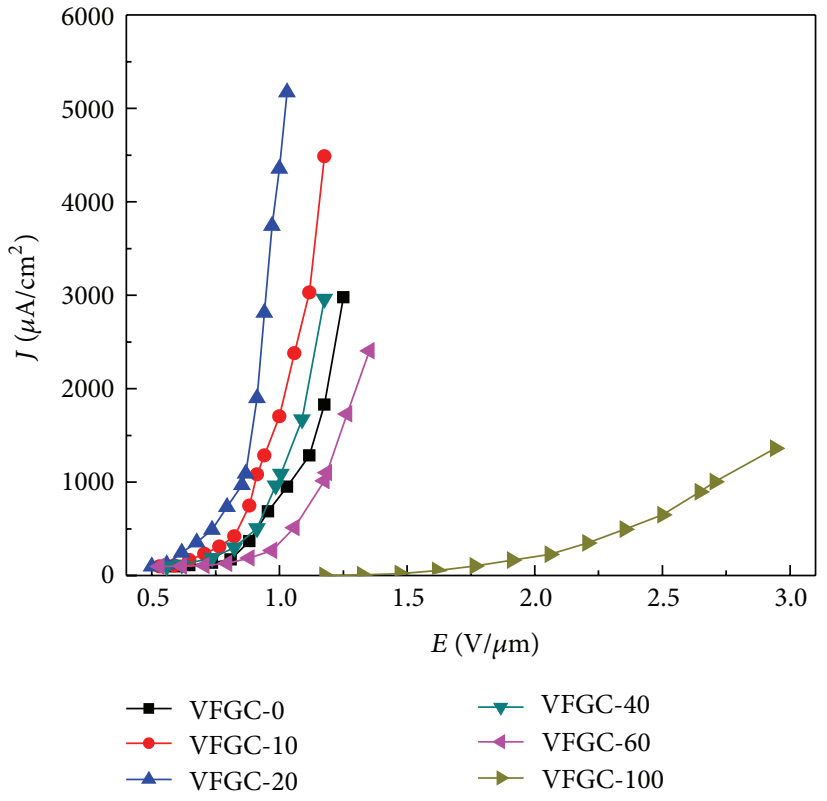

(b)

FIGURE 1: Emission current density versus applied electric field of (a) screen-printed hybrid films of different weight ratios of graphene and (b) vacuum filtered hybrid films of different weight ratios of graphene.

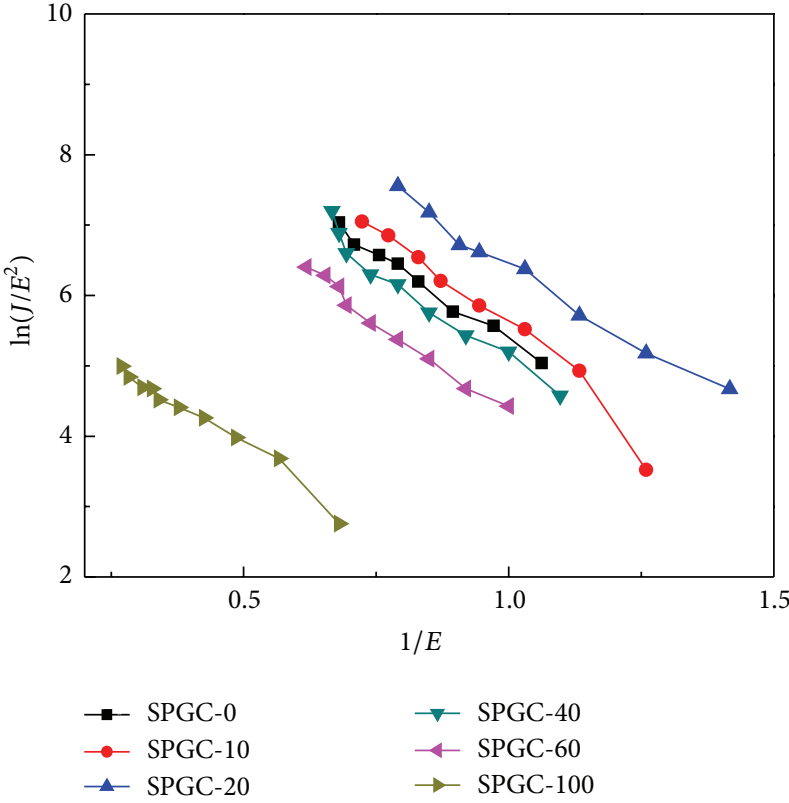

(a)

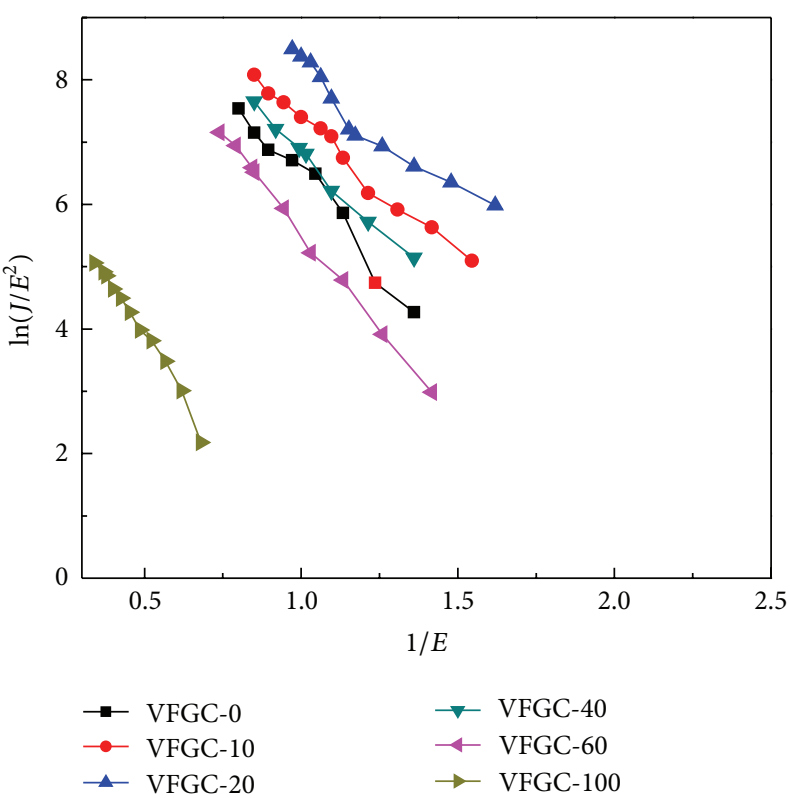

(b)

FIGURE 2: The Fowler-Nordheim (F-N) curves of (a) screen printed samples and (b) vacuum filtered samples.

The reduced F-N equation developed by Forbes [27] indicates the linear relationship between $\ln \left(J / E^{2}\right)$ and $1 / E$ as follows:

$$
\ln \left(\frac{J}{E^{2}}\right)=\frac{-6.3 \times 10^{3} \Phi^{1.5}}{\beta E}+C,
$$

where $J$ is the emission current density, $A=1.56 \times$ $10^{-6} \mathrm{~A} \mathrm{~V}^{-2} \mathrm{eV}, B=6.83 \times 10^{9} \mathrm{eV}^{-3 / 2} \mathrm{~V} \mathrm{~m}^{-1}[3], \beta$ is the field enhancement factor, $\Phi$ is the work function, $E$ is the applied electric field, and $C$ is a constant. Assuming that the work function of the DWCNTs is $5.0 \mathrm{eV}$ [28], we ploted $\ln \left(J / E^{2}\right)$ versus 1/E in Figures 2(a) and 2(b) and made linear fit about 


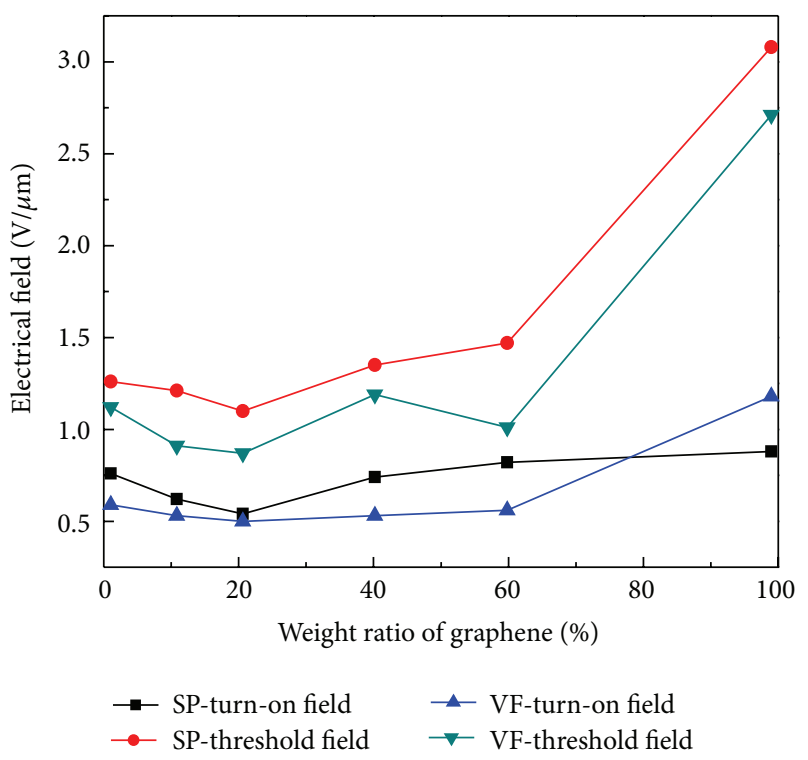

(a)

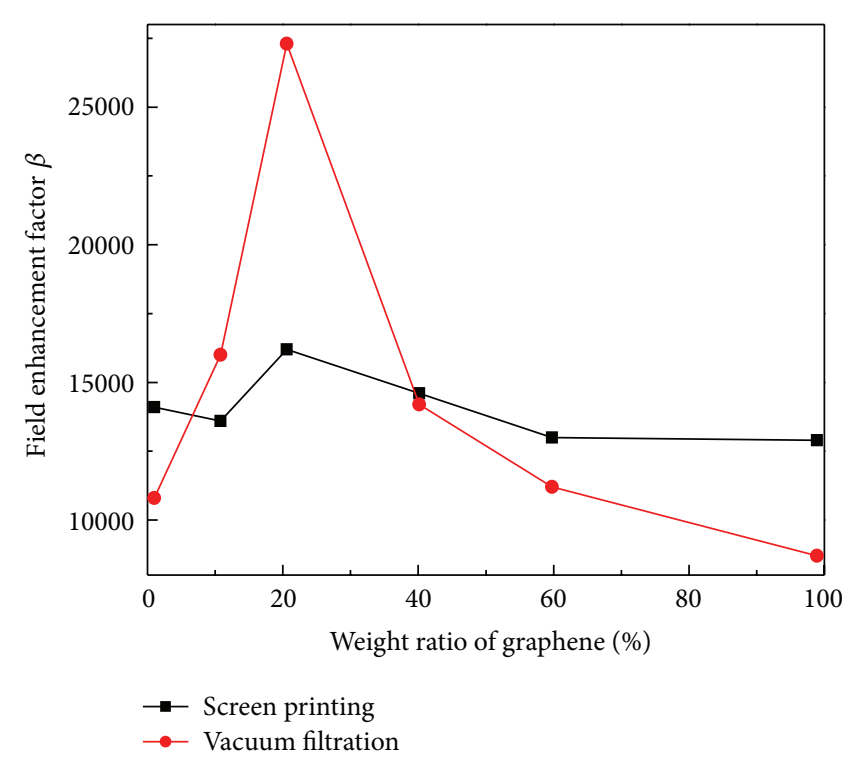

(b)

Figure 3: (a) Turn-on fields and threshold fields of different samples and (b) field enhancement factor of different samples.

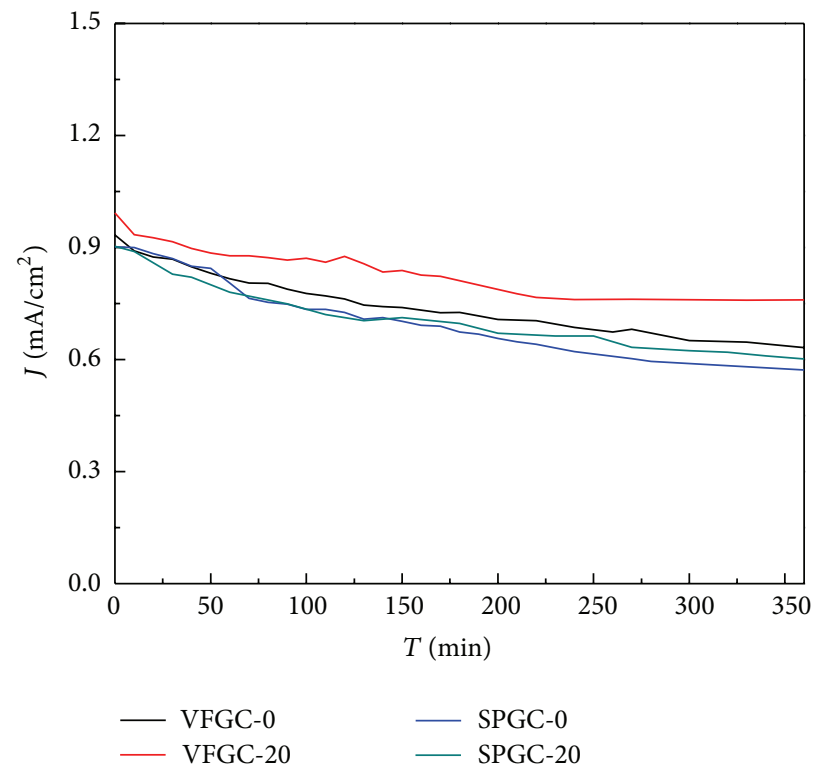

FIGURE 4: The emission current stability of VFGC-0, VFGC-20, SPGC-0, and SPGC-20.

it. According to (2) and the slope of the F-N curve, we could easily calculate the value of $\beta$.

The turn-on field values, threshold field values (Figure 3(a)), and the corresponding enhancement factor values (Figure 3(b)) of different samples with different preparation methods were measured. The electron emission turn-on fields of VFGC samples vary from $0.50 \mathrm{~V} / \mu \mathrm{m}$ to $1.18 \mathrm{~V} / \mu \mathrm{m}$, and they are lower than those of SPGC hybrid samples, where turn-on fields vary from $0.54 \mathrm{~V} / \mu \mathrm{m}$ to $0.88 \mathrm{~V} / \mu \mathrm{m}$, when they have the same weight ratio of graphene. The field enhancement factors of VFGC-0, VFGC10, VFGC-20, VFGC-40, VFGC-60, VFGC-100, SPGC-0,
SPGC-10, SPGC-20, SPGC-40, SPGC-60, and SPGC-100 are $10800,16000,27300,14200,11200,8700,14100,13600$, $16200,14600,13000$, and 12900 , respectively, as shown in Figure 3(b). It is clear that the graphene-DWCNT hybrid films show a better performance than pure DWCNT films. And the VFGC-20 sample obtains the best field emission properties with a turn-on field of $0.50 \mathrm{~V} / \mu \mathrm{m}$, a threshold field of $0.87 \mathrm{~V} / \mu \mathrm{m}$, and an enhancement factor of 27300 .

We evaluated the emission current stability (Figure 4) and the luminance uniformity (Figure 5) of filtered graphene-DWCNT hybrid films and screen-printed grapheneDWCNT hybrid films. The initial emission current density 


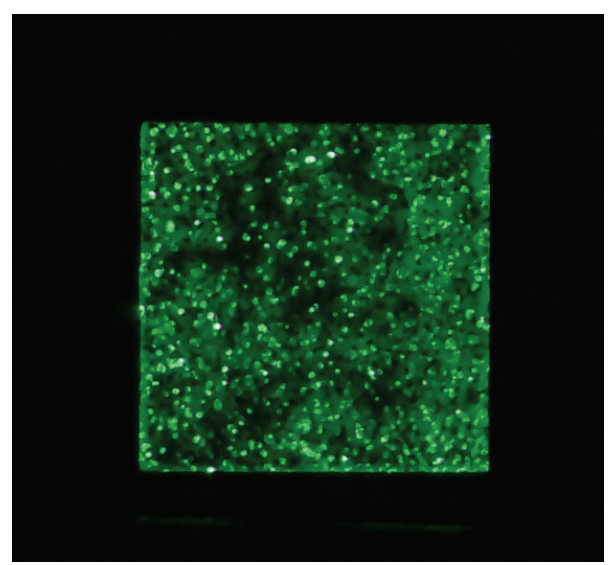

(a)

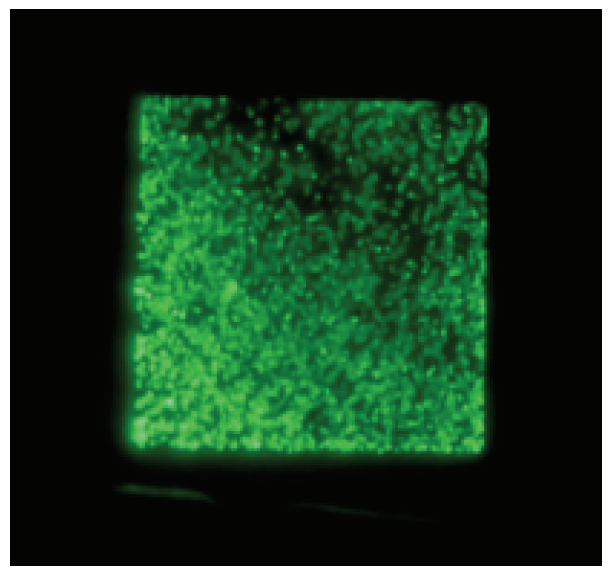

(c)

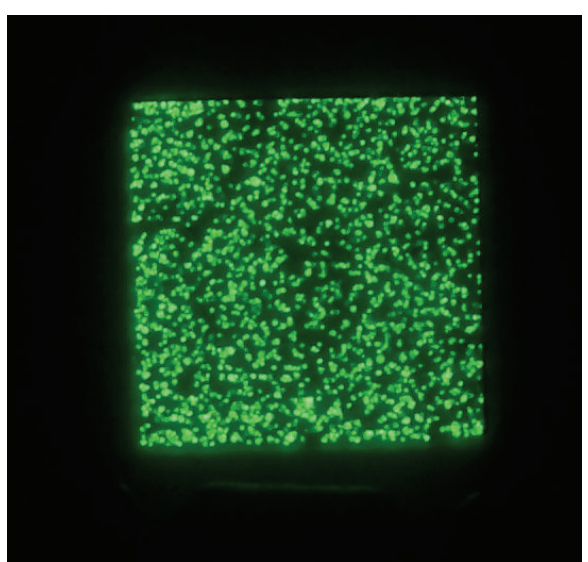

(b)

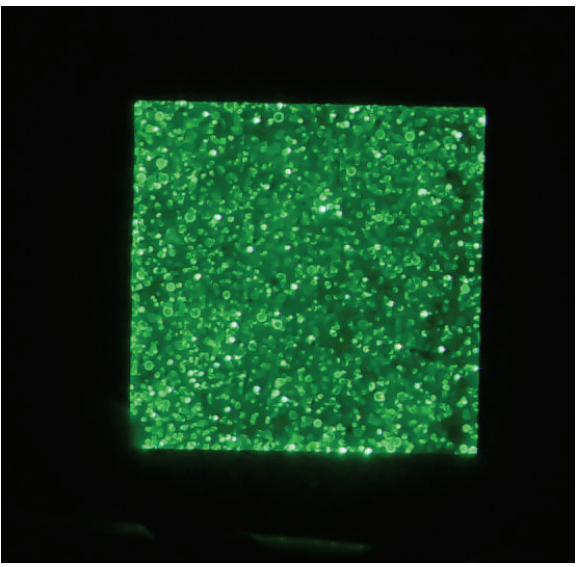

(d)

FIGURE 5: The luminance uniformity of (a) SPGC-0, (b) VFGC-0, (c) SPGC-20, and (d) VFGC-20.

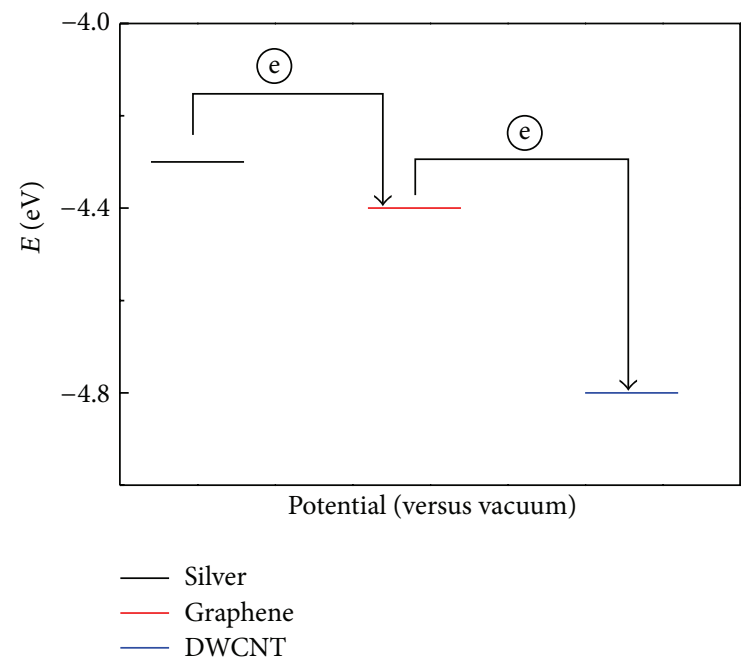

FIGURE 6: Fermi levels of graphene, DWCNT, and silver.

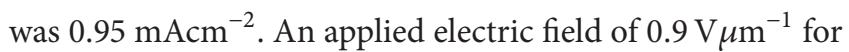

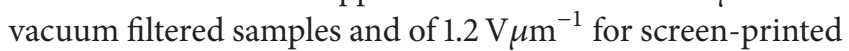

samples was kept constant for $360 \mathrm{~min}$ in the stability test. Figures 4 and 5 demonstrated that the current stability and the luminance uniformity of filtered samples are better than those of screen-printed samples when they have the same weight ratio of graphene. At the same time, hybrid films show better current stability and luminance uniformity than those of the pure DWCNT films prepared by the same method.

It is well known that the process of field emission includes three key steps [29]. First, the electrons transfer from the electrode to the field emission material. Second, the electrons are transmited in the field emission material. At last, the electrons are emitted from the emitter to vacuum.

In the first field emission step, electrons are transported from metal electrode to the nanocarbon hybrid films. Apparently, whether the contact interface between metal electrode and nanocarbon hybrid films is blocked or not would highly affect the sample's emission properties. There are two important factors which affect the electrical contact between metal electrode and hybrid films. One is the Fermi energies of metal and nanocarbon hybrid films. The Fermi energies of silver, graphene, and DWCNTs are $-4.3 \mathrm{eV},-4.4 \mathrm{eV}$, and $-4.8 \mathrm{eV}$, respectively, as shown in Figure 6 [30-32]. Electrons 

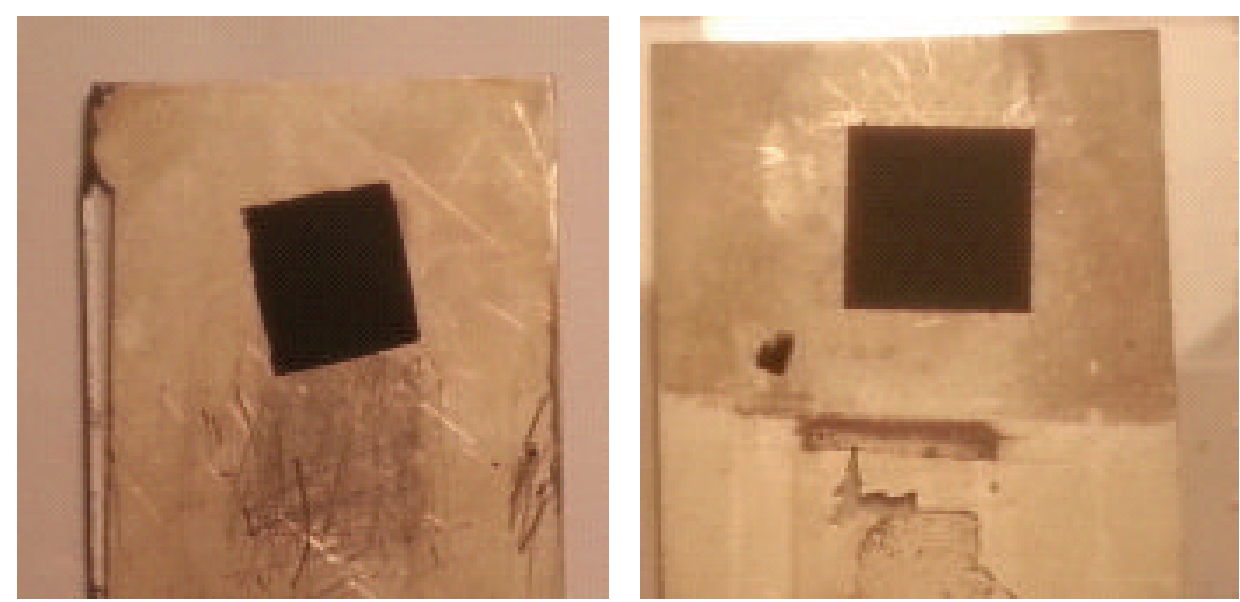

(a)
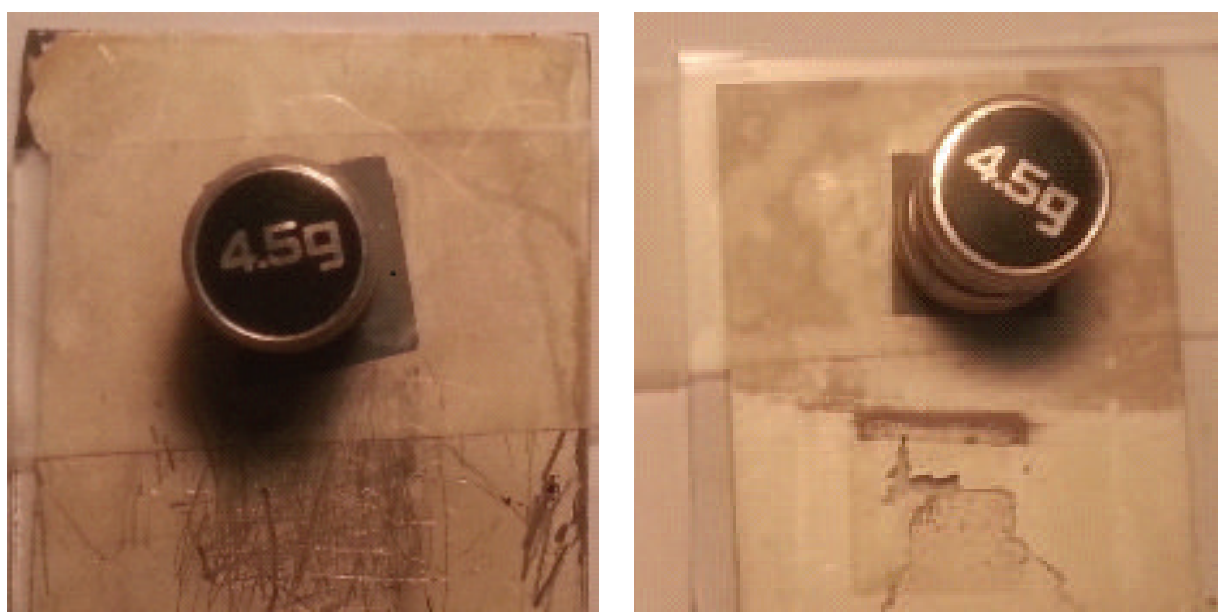

(b)
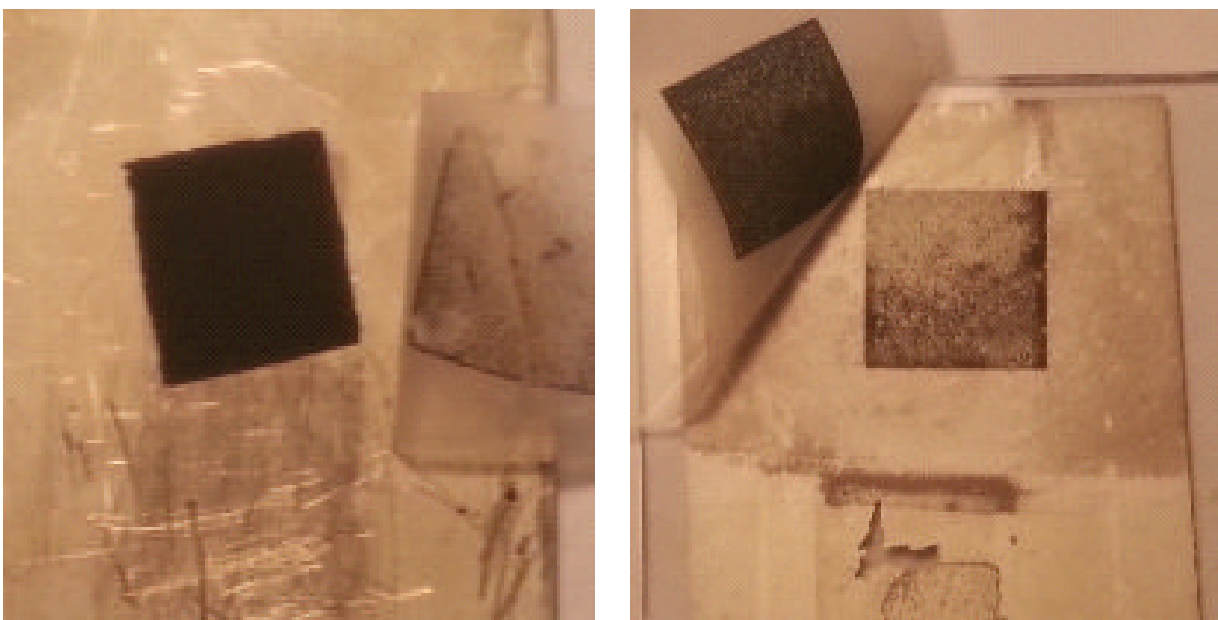

(c)

FIGURE 7: (a) Original vacuum filtered hybrid films (left) and screen-printed hybrid films (right). (b) The same pressure is loaded on adhesive tapes which are attached on hybrid films. (c) Adhesive tapes are peeled off the hybrid films. 


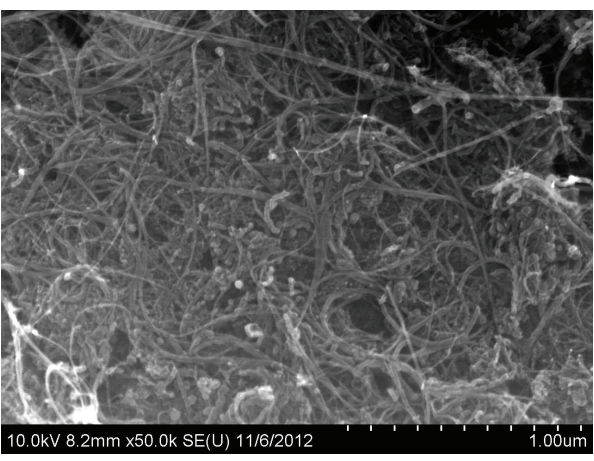

(a)

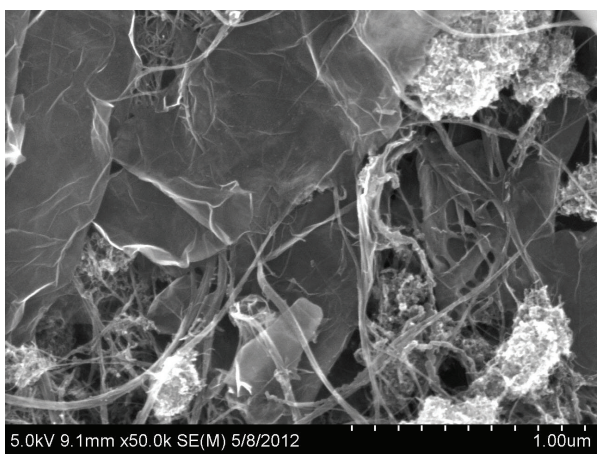

(c)

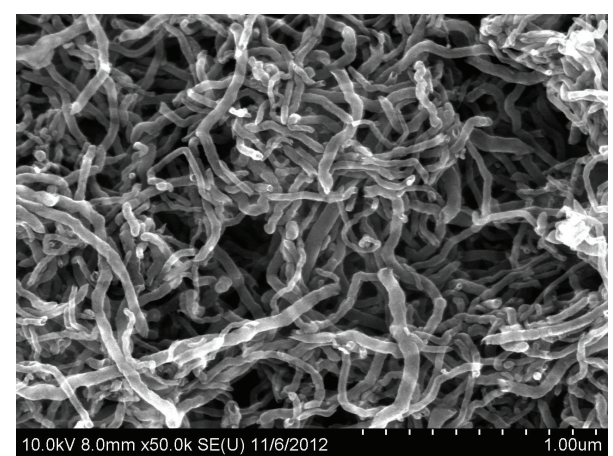

(b)

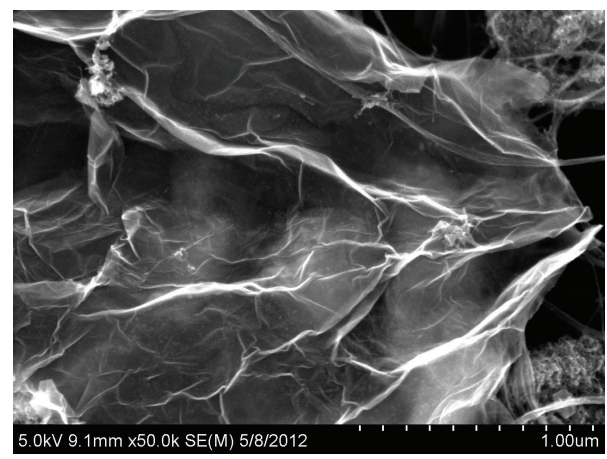

(d)

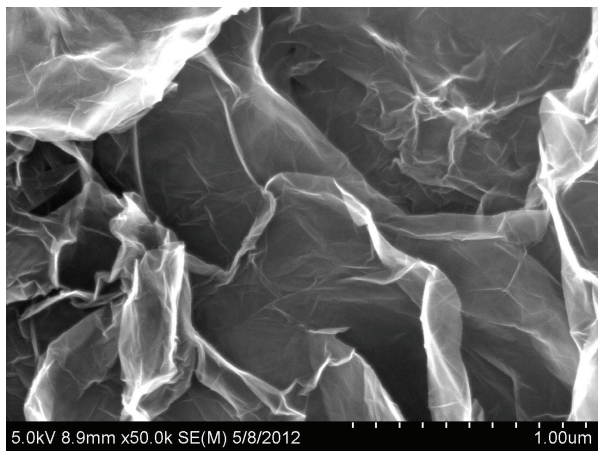

(e)

Figure 8: FESEM images of (a) SPGC-0, (b) VFGC-0, (c) VFGC-20, (d) VFGC-60, and (e) VFGC-100.

can easily transfer from silver films to DWCNT films via graphene.

The other is the adhesive strength of nanocarbon films with metal electrode. The adhesion test was performed by using a Scotch tape based on ASTM standard (D3359-02). The two kinds of DWCNT cathode surface were adhered with the same tapes and loaded with the same pressure $(0.09 \mathrm{~N})$. Figure 7 demonstrated that the adhesive strength of filtered films with silver substrate is stronger than that of screenprinted films with silver substrate.

In the second step, electrons are transported between single DWCNT in the nanocarbon films. In this process, the key factor is the conductivity of nanocarbon films. Figure 8 is the FESEM images of nanocarbon hybrid films.

The conductivity of filtered samples is better than that of screen-printed samples because of the residual organic binders in screen-printed films, and the filtered films are very pure (Figures $8(\mathrm{a})$ and $8(\mathrm{~b})$ ). It is maybe the organic paste used in screen printing process that cannot totally burn out at $300^{\circ} \mathrm{C}$. So the conductivity of screen-printed samples is worse than that of filtered samples. The electrical connection between single carbon nanotube turns better by adding graphene into DWCNTs as the hybrid field emission material (Figure 8(c)).

In the last step, electrons are emitted from DWCNT tips into vacuum. There are two factors which would affect the emission properties: the number of electron emission DWCNT tips and the work function of DWCNT. Some DWCNTs are covered by graphene when the weight ratio of graphene is 20\%, which is shown in Figure 8(c). And almost all DWCNTs are wrapped up by graphene when weight ratio of graphene is $60 \%$; the electron emitting DWCNT tips are 
reduced significantly in Figure 8(d). This is the major reason that the field emission properties are getting worse when the ratio of graphene exceeded 20\%. And Figure 8(e) shows that the edges of graphene are not flat and the surface is not smooth. The zigzag edges and uneven surface are electron emitting tips for graphene films.

\section{Conclusion}

We fabricated the graphene-DWCNT hybrid films by vacuum filtration and screen printing. And we systematically studied the field emission properties of graphene-DWCNT hybrid films with different weight ratios of graphene and with different preparation methods. We found that, compared with screen-printed nanocarbon hybrid films, vacuum filtered nanocarbon hybrid films show lower turn-on field and higher field enhancement factor, and the field emission performance improved significantly when adding graphene into DWCNTs as the emission material. We got the optimum FE performance with a turn-on field of $0.50 \mathrm{~V} \mu \mathrm{m}^{-1}$ and

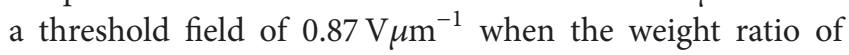
graphene is $20 \%$.

\section{Acknowledgments}

This work was supported by the Rising Star Program of Shanghai under Grant no. 07QA14019, the Shanghai Talent Development Fund, and the Fundamental Research Funds for the Central Universities.

\section{References}

[1] R. H. Fowler and L. Nordheim, "Electron emission in intense electric fields," Proceedings of the Royal Society of London A, vol. 119, pp. 173-181, 1928.

[2] I. Brodie and P. R. Schwoebel, "Vacuum microelectronic devices," Proceedings of the IEEE, vol. 82, no. 7, pp. 1006-1034, 1994.

[3] N. De Jonge and J.-M. Bonard, "Carbon nanotube electron sources and applications," Philosophical Transactions of the Royal Society A, vol. 362, no. 1823, pp. 2239-2266, 2004.

[4] H. J. Kim, J. M. Ha, S. H. Heo, and S. O. Cho, "Small-sized flat-tip CNT emitters for miniaturized X-ray tubes," Journal of Nanomaterials, vol. 2012, Article ID 854602, 6 pages, 2012.

[5] S. Lee, W. B. Im, J. H. Kang, and D. Y. Jeon, "Low temperature burnable carbon nanotube paste component for carbon nanotube field emitter backlight unit," Journal of Vacuum Science and Technology B, vol. 23, no. 2, pp. 745-748, 2005.

[6] S. Roh, J. Lee, M. Jang et al., "Characteristic features of stonewales defects in single-walled carbon nanotube; Adsorption, dispersion, and field emission," Journal of Nanomaterials, vol. 2010, Article ID 398621, 6 pages, 2010.

[7] X. Li, S. Zuo, W. Liu, Y. He, Z. Xiao, and C. Zhu, "Field emission properties of the dendritic carbon nanotubes film embedded with ZnO quantum dots," Journal of Nanomaterials, vol. 2011, Article ID 382068, 5 pages, 2011.

[8] W. A. De Heer, A. Châtelain, and D. Ugarte, "A carbon nanotube field-emission electron source," Science, vol. 270, no. 5239, pp. 1179-1180, 1995.
[9] J. Zhao, J. Zhang, Y. Su, Z. Yang, L. Wei, and Y. Zhang, "Synthesis of straight multi-walled carbon nanotubes by arc discharge in air and their field emission properties," Journal of Materials Science, vol. 47, pp. 6535-6541, 2012.

[10] Y. Saito and S. Uemura, "Field emission from carbon nanotubes and its application to electron sources," Carbon, vol. 38, no. 2, pp. 169-182, 2000.

[11] J. Yu and D. H. C. Chua, "Effective electron emitters by molybdenum oxide-coated carbon nanotubes core-shell nanostructures," Journal of Materials Science, vol. 46, no. 14, pp. 48584863, 2011.

[12] Y. Liu and S. Fan, "Enhancement of field emission properties of cyanoacrylate-carbon nanotube arrays by laser treatment," Nanotechnology, vol. 15, no. 8, pp. 1033-1037, 2004.

[13] B.-R. Huang, Y.-K. Yang, T.-C. Lin, and W.-L. Yang, "Core-shell structure of a silicon nanorod/carbon nanotube field emission cathode," Journal of Nanomaterials, vol. 2012, Article ID 369763, 6 pages, 2012.

[14] M. Mohammad, M. B. Khan, T. A. Sherazi, J. Anguita, and D. Adikaari, "Fabrication of vertically aligned CNT composite for membrane applications using chemical vapor deposition through In Situ polymerization," Journal of Nanomaterials, vol. 2013, Article ID 713583, 5 pages, 2013.

[15] G. F. Malgas, C. J. Arendse, N. P. Cele, and F. R. Cummings, "Effect of mixture ratios and nitrogen carrier gas flow rates on the morphology of carbon nanotube structures grown by CVD," Journal of Materials Science, vol. 43, no. 3, pp. 1020-1025, 2008.

[16] T. Feng, L. Dai, J. Jiang et al., "Memory emission of printed carbon nanotube cathodes," Applied Physics Letters, vol. 88, Article ID 203108, 2006.

[17] L. Wang, Y. Chen, T. Chen, W. Que, and Z. Sun, “Optimization of field emission properties of carbon nanotubes cathodes by electrophoretic deposition," Materials Letters, vol. 61, no. 4-5, pp. 1265-1269, 2007.

[18] Z. Wu, Z. Chen, X. Du et al., "Transparent, conductive carbon nanotube films," Science, vol. 305, no. 5688, pp. 1273-1276, 2004.

[19] J. Zhang, X. Wang, W. Yang et al., "Interaction between carbon nanotubes and substrate and its implication on field emission mechanism," Carbon, vol. 44, no. 3, pp. 418-422, 2006.

[20] A. K. Geim and K. S. Novoselov, "The rise of graphene," Nature Materials, vol. 6, no. 3, pp. 183-191, 2007.

[21] W. Choi, I. Lahiri, R. Seelaboyina, and Y. S. Kang, "Synthesis of graphene and its applications: a review," Critical Reviews in Solid State and Materials Sciences, vol. 35, no. 1, pp. 52-71, 2010.

[22] I. Lahiri, V. P. Verma, and W. Choi, "An all-graphene based transparent and flexible field emission device," Carbon, vol. 49, no. 5, pp. 1614-1619, 2011.

[23] B. B. Wang, Q. J. Cheng, X. Chen, and K. Ostrikov, "Enhancement of electron field emission of vertically aligned carbon nanotubes by nitrogen plasma treatment," Journal of Alloys and Compounds, vol. 509, no. 38, pp. 9329-9334, 2011.

[24] J. J. Wang, M. Y. Zhu, R. A. Outlaw et al., "Free-standing subnanometer qraphite sheets," Applied Physics Letters, vol. 85, no. 7, pp. 1265-1267, 2004.

[25] A. N. Obraztsov, A. V. Tyurnina, E. A. Obraztsova et al., "Raman scattering characterization of CVD graphite films," Carbon, vol. 46, no. 6, pp. 963-968, 2008.

[26] S. Sethi and A. Dhinojwala, "Superhydrophobic conductive carbon nanotube coatings for steel," Langmuir, vol. 25, no. 8, pp. 4311-4313, 2009. 
[27] R. G. Forbes, "Simple good approximations for the special elliptic functions in standard Fowler-Nordheim tunneling theory for a Schottky-Nordheim barrier," Applied Physics Letters, vol. 89, Article ID 113122, 2006.

[28] N. De Jonge, M. Allioux, M. Doytcheva et al., "Characterization of the field emission properties of individual thin carbon nanotubes," Applied Physics Letters, vol. 85, no. 9, pp. 1607-1609, 2004.

[29] J. Xu, R. Pan, Y. Chen et al., "Electron field emission from screen-printed graphene/DWCNT composite films," Journal of Alloys and Compounds, vol. 551, pp. 348-351, 2012.

[30] R. Czerw, B. Foley, D. Tekleab, A. Rubio, P. M. Ajayan, and D. L. Carroll, "Substrate-interface interactions between carbon nanotubes and the supporting substrate," Physical Review B, vol. 66, no. 3, Article ID 033408, 2002.

[31] Y. Yao, G. Li, S. Ciston, R. M. Lueptow, and K. A. Gray, "Photoreactive $\mathrm{TiO}_{2} /$ carbon nanotube composites: synthesis and reactivity," Environmental Science and Technology, vol. 42, no. 13, pp. 4952-4957, 2008.

[32] M. Chelvayohan and C. H. B. Mee, "Work function measurements on (110), (100) and (111) surfaces of silver," Journal of Physics C, vol. 15, no. 10, article 029, pp. 2305-2312, 1982. 

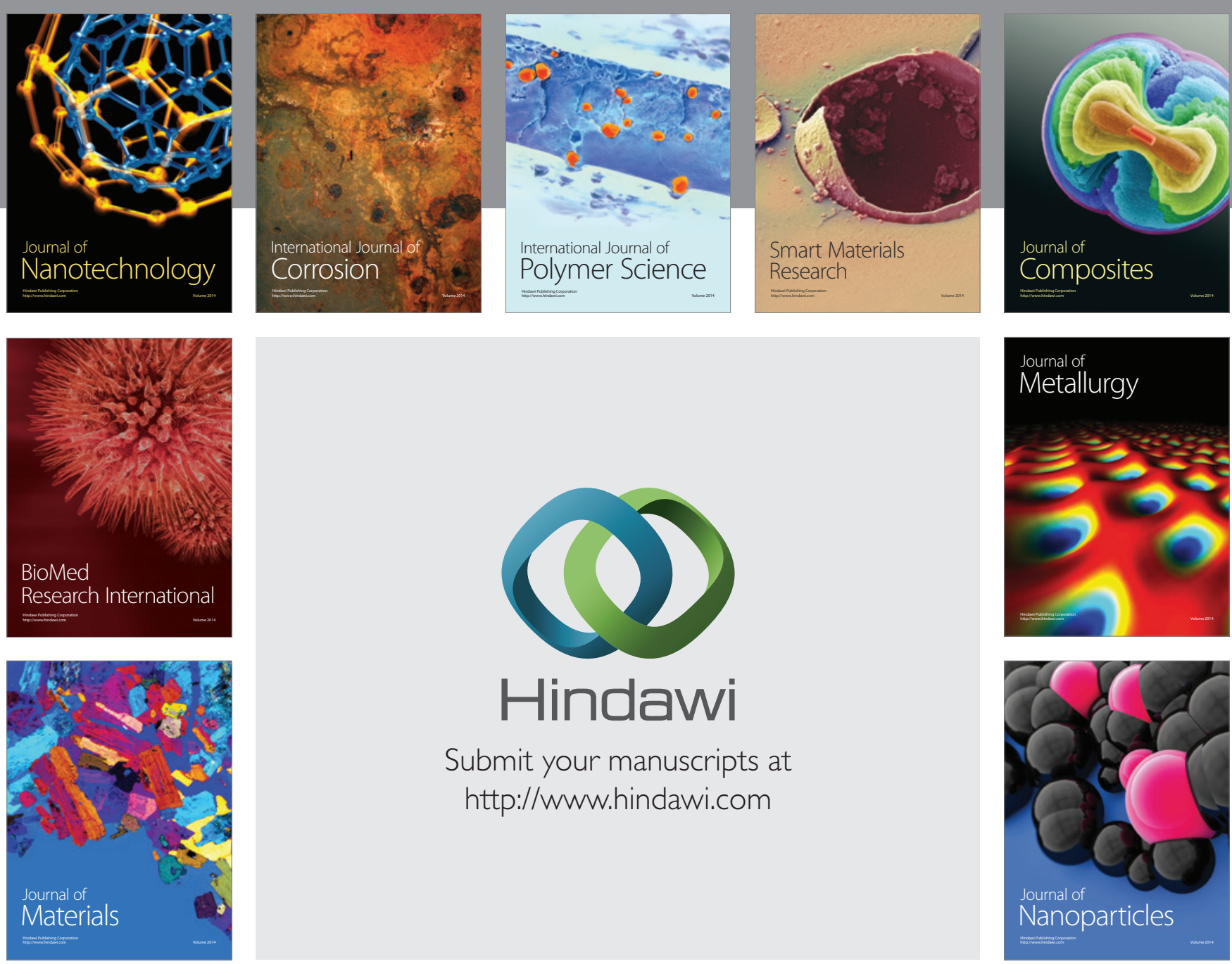

Submit your manuscripts at http://www.hindawi.com
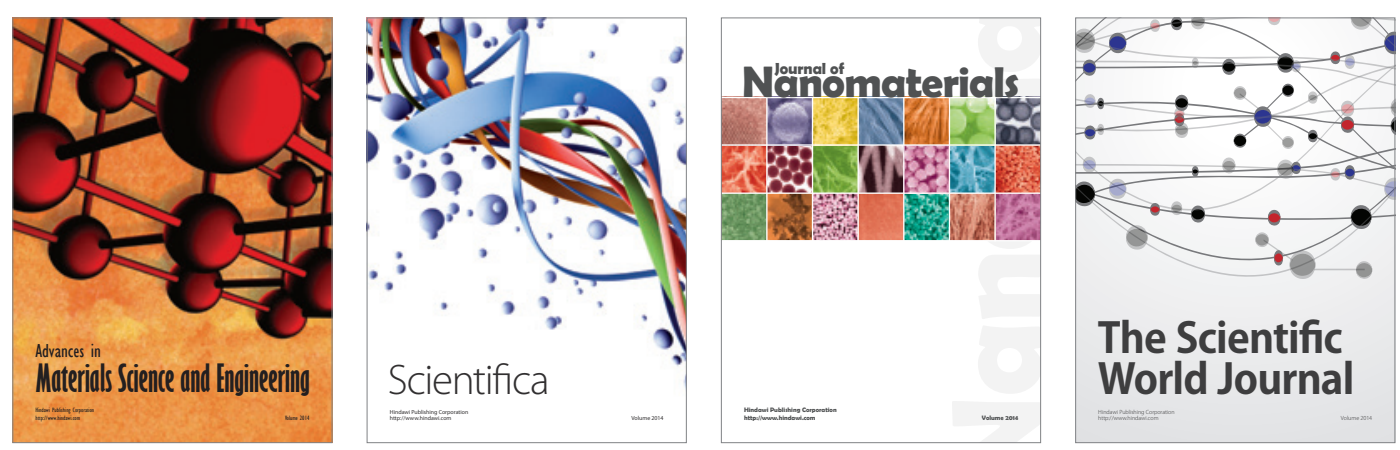

\section{The Scientific World Journal}
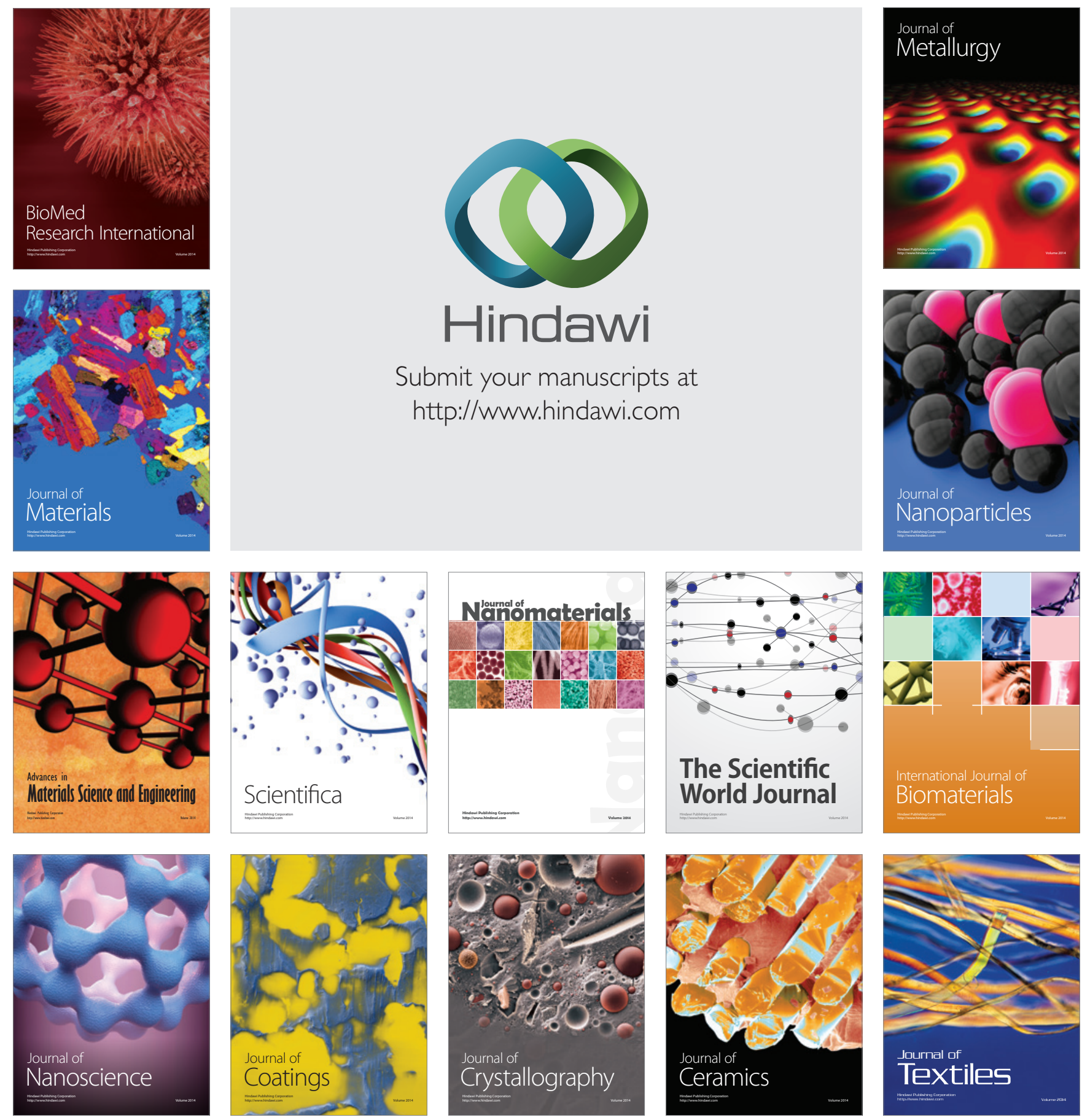